\title{
Einige praktische Hinweise im Falle eines Behandlungsfehlers
}

Ärzte sehen sich durch ihre Patienten immer häufiger mit dem Vorwurf eines ärztlichen Behandlungsfehlers konfrontiert. Dieser Artikel soll die wichtigsten Verhaltensregeln in Erinnerung rufen.
Zunächst ist entscheidend, dass dem Haftpflichtversicherer gegenüber unverzüglich eine schriftliche Schadensanzeige erfolgt. Nach § 153 Versicherungsvertragsgesetz (VVG) hat der Versicherungsnehmer innerhalb einer Woche die Tatsachen anzuzeigen, die seine Verantwortlichkeit gegenüber einem Dritten zur Folge haben könnten. Die nicht rechtzeitige Schadensanzeige stellt eine Obliegenheitsverletzung aus dem Versicherungsvertrag dar und kann schlimmstenfalls dazu führen, dass der Versicherer von seiner Leistungspflicht frei bleibt. Die Obliegenheit zur Schadensmeldung besteht unabhängig davon, ob die geltend gemachten Ansprüche aus Sicht des Arztes berechtigt sind oder nicht. Der Schadensmeldung ist eine möglichst umfassende Darstellung des Behandlungsgeschehens sowie eine Stellungnahme zu den erhobenen Vorwürfen beizufügen.

Der Arzt, dem ein Behandlungsfehler vorgeworfen wird, sollte sich unbedingt davor hüten, vor der Schadensanzeige an den Versicherer dem Patienten oder dessen Bevollmächtigten gegenüber seine Haftung anzuerkennen oder gar bezifferte Schadensersatz- bzw. Schmerzensgeldforderungen zu befriedigen. Die Versicherung muss hierzu ihre Zustimmung erklären, anderenfalls kann sie sich von ihrer Leistungspflicht befreien.

\section{Außergerichtliche Lösung suchen}

Eine Vielzahl von Haftpflichtprozessen wird nur deshalb geführt, weil beim ersten Vorwurf eines Behandlungsfehlers die Fronten sogleich derart verhärten, dass nur noch mit gerichtlicher Hilfe ein Interessenausgleich erfolgen kann. Die Ursachen hierfür sind mannigfaltig, vor allem aber wohl darin zu sehen, dass der Gegenstand des Vorwurfs, die Beeinträchtigung der Gesundheit, des Menschen höchstes Gut ist. Die Patienten führen ihre Klage mit hoher Emotionalität. Der angegriffene Arzt dagegen blockt nicht selten $a b$, da der Vorwurf seinem ärztlichen Selbstverständnis zuwiderläuft, das darin besteht, die Gesundheit des einzelnen Menschen zu schützen und wieder herzustellen, Leben zu erhalten, Leiden zu lindern.

Der erste Streit wird dann oftmals schon um das Begehren des Patienten geführt, Einsicht in die ihn betreffenden vollständigen Behandlungsunterlagen zu erlangen. Hierüber verbietet sich aber letztlich jeder Streit: Der Patient hat grundsätzlich einen Anspruch auf Einsichtnahme bzw. Herausgabe seiner Krankenunterlagen (Kopien gegen Kostenersatz). Mit Zustimmung des Patienten ist aber auch die Herausgabe an Dritte, wie z.B. einen Rechtsanwalt, ohne Weiteres zulässig. Ein Arzt, der hier schon mauert, nährt den Verdacht, es gebe etwas zu verbergen und riskiert die klageweise Geltendmachung des Einsichtnahme- bzw. Herausgabeanspruchs. Dieser Prozess geht regelmäßig zu Lasten des Arztes aus. Es empfiehlt sich unbedingt, auf Verlangen des Patienten, Einsicht in die ihn betreffenden Unterlagen zu gewähren. Allerdings ist darauf zu achten, dass ihm solche Aufzeichnungen aus den Behandlungsunterlagen zulässigerweise vorenthalten werden dürfen, die persönliche 
subjektive Bewertungen des Arztes über dessen Person oder Krankheitsgeschehen enthalten, wie z.B. „Patient ist Simulant/Querulant“.

Schlichtung vorschlagen

Dem anspruchstellenden Patienten sollte nach Rücksprache mit der Haftpflichtversicherung ein Schlichtungsverfahren vor den bei den Landesärztekammern angesiedelten Gutachter- und Schlichtungsstellen vorgeschlagen werden. Dabei begutachten unabhängige Sachverständige das Behandlungsgeschehen. Das Verfahren ist kostenfrei. Der von der Gutachterund Schlichtungsstelle erlassene Bescheid kann von den Beteiligten im Wege des Widerspruchs angefochten werden mit dem Ziel, eine weitere Begutachtung durch die Gutachterkommission bei der Landesärztekammer durchführen zu lassen. Der Schlichterspruch bindet die Beteiligten nicht, so dass unabhängig davon stets auch der Klageweg zu den Zivilgerichten eröffnet ist.

\section{Klage als Ultima ratio}

Lässt sich der Behandlungsfehlervorwurf trotz aller Bemühungen nicht außergerichtlich klären, so wird der Patient möglicherweise Klage auf Schadensersatz und Schmerzensgeld erheben. Je nach Höhe des Streitwerts ist entweder das Amtsgericht (derzeit bis 10000 DM ) oder das Landgericht (ab 10001 DM) zuständig.

Die Prozessführung muss der Arzt grundsätzlich seiner Haftpflichtversicherung überlassen, um deren Geld es letztendlich in dem Verfahren geht. So hat auch nur sie zu entscheiden, ob sie den Anspruch anerkennt, dem Patienten einen Vergleich anbietet oder die Angelegenheit ausficht. Regelmäßig beauftragen die Haftpflichtversicherer entsprechend versierte Rechtsanwälte mit der Prozessvertretung. Sofern Klage beim Landgericht erhoben ist, müssen sie einen Anwalt beauftragen, weil vor diesen Gerichten Anwaltszwang herrscht. Manche Versicherungsgesellschaften arbeiten mit Vertrauensanwälten zusammen. Andere stellen es ihren Versicherungsnehmern frei, von welchem Anwalt sie vertreten werden möchten. Man sollte also nicht ohne Rücksprache mit der Versicherung einen Anwalt seiner Wahl beauftragen, schließlich zahlt diese auch das Anwaltshonorar. Zieht der Arzt neben dem von der Versicherung beauftragten einen weiteren Anwalt hinzu, so geht dieser Luxus auf Kosten des Versicherungsnehmers.

Die Gerichtsverfahren ziehen sich in aller Regel über einen langen Zeitraum, manchmal über Jahre, hin, wenn es nicht - was zu jedem Zeitpunkt möglich ist - zu einer Verständigung zwischen den Parteien kommt. Die lange Verfahrensdauer beruht darauf, dass von Gerichts wegen in aller Regel Beweis durch ein unabhängiges Sachverständigengutachten erhoben wird. Die Suche nach einem Gutachter gestaltet sich zuweilen kompliziert. Sei es, dass die Parteien Einwändungen gegen die Auswahl des Gutachters vorbringen, oder dass alle geeignet erscheinenden Gutachter den Gutachtenauftrag zurückgeben bzw. mitteilen lassen, dass aufgrund beruflicher Belastungen mit einer Gutachtenerstattung nicht vor Ablauf eines halben Jahres gerechnet werden dürfe. Aber selbst dann, wenn endlich das Gutachten vorliegt, kann auf Antrag oder von Amts wegen ein Ergänzungs- oder Obergutachten eingeholt werden, was dann wiederum den Prozess enorm verzögert. Man darf sich keinen Illusionen hingeben: Der Ausgang des Verfahrens hängt nicht von der argumentativen Überzeugungskraft der Parteien oder dem richterlichen Dafürhalten ab, sondern allein von dem, was der Gutachter sagt. Hieran wird sich das Gericht mangels eigener Sachkunde orientieren.
Arzthaftpflichtprozesse sind außerordentlich unerfreulich. Sie verhelfen dem Arzt oder Patienten manchmal erst nach Jahren zu seinem Recht, was von beiden Seiten als unzumutbar angesehen wird. Deshalb sei empfohlen, sich nach Kräften um eine außergerichtliche Beilegung der Streitigkeiten zu bemühen.

Constantin Frh. Droste zu Senden, Wiesbaden

c/o Rechtsanwälte Broglie, Schade \& Partner, Sonnenberger Str. 16, 65193 Wiesabden, Tel. 0611/180950, Fax 0611/18059518 